\title{
An Arginine to Glutamine Mutation in Residue 109 of Human Ornithine Transcarbamylase Completely Abolishes Enzymatic Activity in Cos1 Cells
}

\author{
Jeannie T. Lee and Robert L. Nussbaum \\ Howard Hughes Medical Institute, Department of Human Genetics, University of Pennsylvania, Philadelphia, Pennsylvania 19104
}

\begin{abstract}
Ornithine transcarbamylase (OTC) is an important enzyme in the detoxification of ammonia to urea, and its deficiency is the most common inborn error of ureagenesis in humans. Among 24 cases of OTC deficiency previously examined, three unrelated individuals all showed loss of a Taq I site in the OTC gene corresponding to codon 109, suggesting that this Taq I site may be prone to mutation. Two of these patients demonstrated the same $C \rightarrow T$ transition (in antisense strand) converting Arg109 to Gln. Although these studies implied a strong association between the missense mutation and OTC-deficient phenotype, a causal relationship could not be firmly established. We have investigated this relationship by reconstructing the mutation in vitro. A full-length human OTC cDNA was cloned into an SV40-based expression vector and has been reproducibly expressed at high levels in the cell line Cos1. By site-directed mutagenesis of this wild type sequence, we constructed a missense mutation which contains the $\mathbf{C} \rightarrow \mathrm{T}$ transition. Electroporation and transient assay in Cos1 indicated that the specific activity of mutant OTC was 100 -fold lower than that of wild type. This result confirms that the Taq I alteration leading to the Gln missense is responsible for the OTC deficiency affecting the above patients.
\end{abstract}

\section{Introduction}

Ornithine transcarbamylase (OTC) ${ }^{1}$ is a urea cycle enzyme which condenses carbamyl phosphate and ornithine to form citrulline. A homotrimer of $36-\mathrm{kD}$ subunits, OTC is localized to the mitochondrial matrix and is found exclusively in two tissues: the liver and small intestine. With the recent cloning of the human OTC gene, the gene was found to be a $73-\mathrm{kb}$ gene containing 10 exons (1).

An X-linked disease, a deficiency of OTC is the most common inborn error of ureagenesis in humans and is a disease with frequent new mutations $(2,3)$. Over 100 cases of OTC deficiency have been examined and documented. This condition results in the inability to detoxify ammonia and ranges in

Address reprint requests to Dr. Robert L. Nussbaum, Howard Hughes Medical Institute, Department of Human Genetics, University of Pennsylvania, 37th St and Hamilton Walk, Philadelphia, PA 19104. Received for publication 29 June 1989.

1. Abbreviations used in this paper: OTC, ornithine transcarbamylase; PAP, placental alkaline phosphatase.

J. Clin. Invest.

(C) The American Society for Clinical Investigation, Inc. $0021-9738 / 89 / 12 / 1762 / 05 \quad \$ 2.00$

Volume 84, December 1989, 1762-1766 severity from neonatal lethality to mild hyperammonemia with developmental delay. It is believed that the severity of symptoms correlates with the nature of the molecular defect (4-7). Biochemical studies on liver biopsy samples have indicated that the disease can result from either quantitative changes (decreased production of OTC) or qualitative changes (altered ligand affinity or catalytic potential) in the enzyme. Unfortunately, few attempts have been made to correlate structural defects with clinical (functional) defect.

With the availability of cDNA probes for OTC, examination of $\mathrm{OTC}^{-}$individuals has shifted to the DNA level. Abnormalities have been found to range from gross gene deletions (8-11) to small mutations detected only with restriction fragment length alterations $(2,3)$. We had previously observed that point mutations constitute a class of OTC mutations that are not only variable in location but also recurrent in independent families $(2,3)$. Among 24 cases examined, three unrelated individuals all showed loss of the same Taq I site corresponding to codon 109. Furthermore, two of these patients demonstrated the same $\mathrm{C} \rightarrow \mathrm{T}$ transition (antisense), converting Arg 109 to Gln. Although strongly suggestive, these observations could not firmly establish a causal relationship between the missense mutation and OTC deficiency. The impact of this mutation is of particular interest because it is generally believed that Arg109 does not reside in either the ornithine- or the carbamyl-phosphate-binding sites. In an attempt to understand molecular structure in relationship to the spectrum of clinical disease, we have reconstructed the Arg to Gln mutation in vitro by site-directed mutagenesis and have subsequently shown that the missense mutation lacks any OTC activity when expressed in tissue culture.

\section{Methods}

\section{Molecular biology}

All techniques in recombinant DNA technology were performed according to Maniatis et al. (12).

\section{Plasmids and oligonucleotide}

The oligonucleotide used in mutagenesis is an 18-mer containing the sequence TATACACTTGAGCCAATA. It is the antisense sequence derived from basepair 317-334 of the published OTC CDNA sequence (13) but contains the $\mathrm{C} \rightarrow \mathrm{T}$ transition at basepair 325 converting Arg to Gln (underlined nucleotide).

pHOC3 contains a 1.5-kb OTC insert originally isolated from a human liver cDNA $\lambda$ gt 11 library (2). pSV2a-dHFR is a ColE1 plasmid containing an SV40 origin of replication and SV40 early promoter and polyadenylation site flanking the dihydrofolate reductase cDNA (14). pSV2-PAP is the placental alkaline phosphatase gene cloned into the above pSV40 backbone (15).

\section{Site-directed mutagenesis and DNA sequencing}

Plasmids, reagents, and protocol used in the mutagenesis were all obtained from Bio-Rad Laboratories, Richmond, CA (Mutagene M13 in 
vitro mutagenesis kit). The oligonucleotide used is described above. DNA sequencing was performed using the new Sequenase technology and ${ }^{35}$ S-labeled adenine nucleotides from standard M13 primers from New England Biolabs, Beverly, MA.

\section{DNA transfection for transient expression}

Cosl is an immortalized African green monkey cell line which is permissive for SV40 replication (16) and was used for all DNA transfections. DNAs were introduced by electroporation for transient assay. The electroporation protocol was modified from Chu et al. (17). Cells were grown to $70 \%$ confluence T-75 flasks in DME/10\% FCS, washed in $1 \times$ PBS, harvested by trypsin digestion, and resuspended in $1 \times$ HBSS at a concentration of $5 \times 10^{5} .80 \mu \mathrm{g}$ of OTC DNA and $5 \mu \mathrm{g}$ of placental alkaline phosphatase (PAP) DNA were added to each cuvette. Electroporation was done in $1-\mathrm{ml}$ cuvettes with platinum electrodes at $175 \mathrm{~V}$. After a 3-d incubation period in DME/10\% FCS, cells were prepared for enzymatic assays as described below.

\section{Enzymatic assays for OTC and PAP}

After transient expression, cells were harvested by scraping with rubber policemen, washing in $1 \times$ PBS, resuspending in $1 \mathrm{ml} 1 \times$ PBS, and aliquoted as follows: $200 \mu \mathrm{l}$ for PAP assay, $700 \mu \mathrm{l}$ for OTC assay, and $100 \mu \mathrm{l}$ for Bradford assay. Each enzymatic assay is a colorimetric assay performed as described below.

$P A P$ assay (15). $200 \mu \mathrm{l}$ of cells were spun down and resuspended in $20 \mu \mathrm{l}$ of $50 \mathrm{mM}$ Tris-pH $7.5,1 \mathrm{mM} \mathrm{MgCl} 2.10 \mu \mathrm{l}$ of cells were heated to $65^{\circ} \mathrm{C}$ for $30 \mathrm{~min}$, added to $5 \mathrm{mM} \mathrm{p}$-nitro-phenyl phosphate substrate reaction mixture, and incubated for $30 \mathrm{~min}$ at $30^{\circ} \mathrm{C}$. PAP activity was then determined from spectrophotometric measurements at $405 \mathrm{~nm}$.

OTC assay (18). $700 \mu \mathrm{l}$ of cells microfuged and resuspended in 200 $\mu \mathrm{l}$ of mitochondrial lysis buffer $(0.5 \%$ Triton, $10 \mathrm{mM}$ Hepes, $2 \mathrm{mM}$ DTT). Lysis was done by sonication in the W375 Ultrasonic Processor (Heat Systems, Ultrasonics, Inc., Farmingdale, NY) at maximal power for $2 \mathrm{~min}$ at $4^{\circ} \mathrm{C}$, and then incubated on ice for $30 \mathrm{~min}$. $50 \mu \mathrm{l}$ of homogenate was then added to a solution of ornithine and triethanolamine to a final volume of $675 \mu$ l. OTC reactions were begun by adding carbamyl phosphate ( $75 \mu \mathrm{l}$ of $150 \mathrm{mM}$ stock). Final concentrations of each reagent were: $5 \mathrm{mM}$ ornithine, $15 \mathrm{mM}$ carbamyl phosphate, and $270 \mathrm{mM}$ triethanolamine. Reactions were stopped by $375 \mu \mathrm{l}$ of 3:1 phosphoric acid/sulfuric acid (by volume). Citrulline production was then determined by adding $47 \mu 13 \%$ 2,3-butanedionemonoxime, boiling in the dark for $15 \mathrm{~min}$, and reading at A-490 $\mathrm{nm}$. The positive control used in these OTC assays is a $2-\mu$ l sample of rat liver mitochondrial homogenate as described in (18).
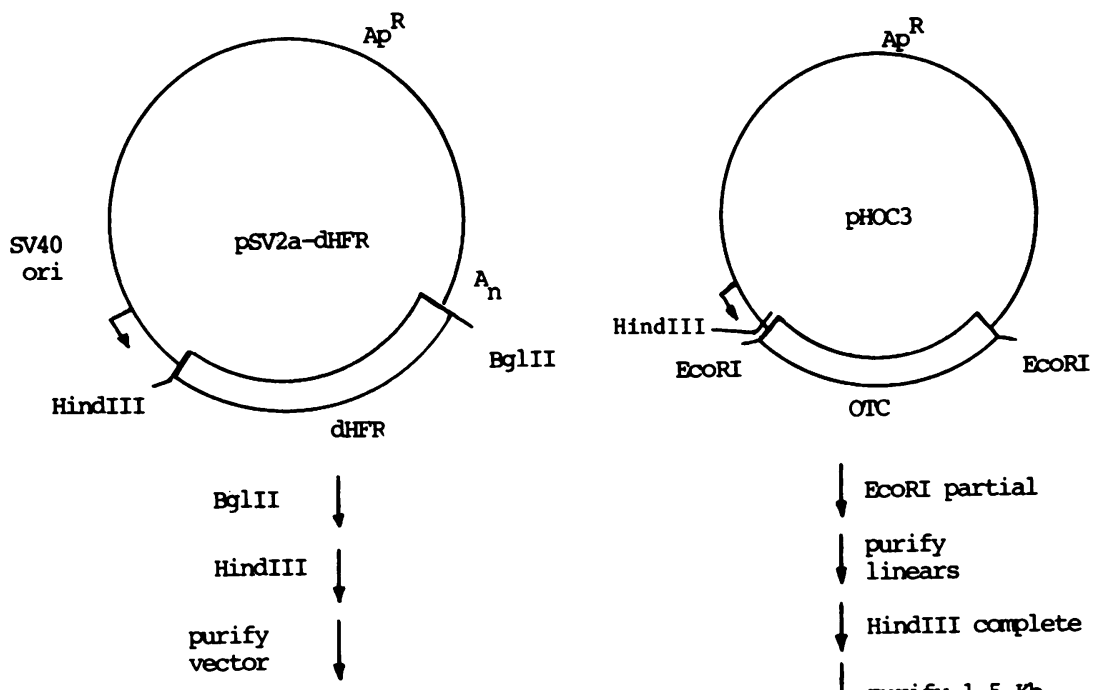

ECORI partial purify linears HindIII complete purify $1.5 \mathrm{~Kb}$ CDNA

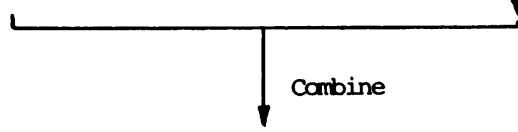

I Add BamHI-ECORI

adaptors

3-piece ligation

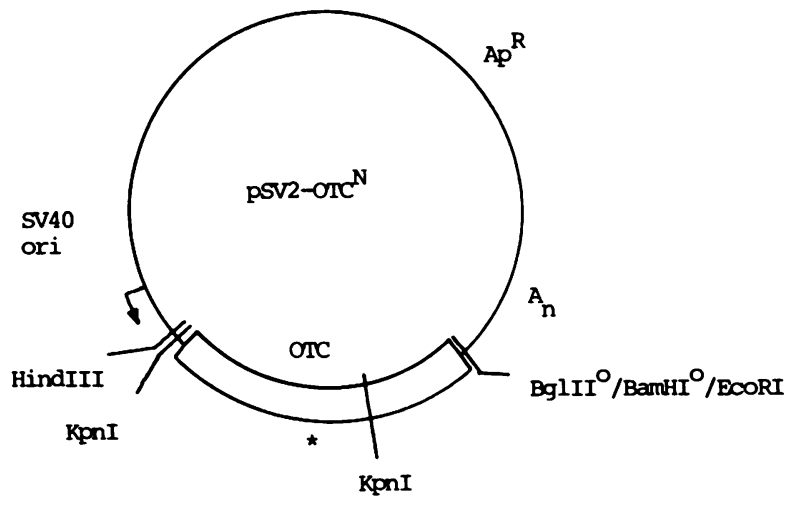

Figure 1. Cloning of OTC cDNA into an SV40-based expression vector. The OTC cDNA was excised from pHOC3, a clone derived from the human liver $\lambda$ gt 11 library (2). pHOC 3 was partially digested with Eco RI. Linearized species were electrophoresed into an agarose gel, isolated on NA45 paper, and then digested with Hind III. The complete OTC cDNA could be identified as a $1.5-\mathrm{kb}$ fragment and was then purified by gel electrophoresis. The pSV2a vector was prepared by excising the dHFR gene from the parent pSV2a-dHFR by digesting with Bgl II and Hind III and purifying the vector backbone. Then the vector and cDNA were combined with Bam HI-Eco RI adaptors in a three-piece ligation reaction to create $\mathrm{pSV} 2-\mathrm{OTC}^{\mathrm{N}}$. The pSV2a vector is derived from pBR322 and SV40. It contains ColE1 origin of replication, the resistance marker for ampicillin, the SV40 origin of replication, early promoter, and the polyadenylation site. $L$ designates the transcriptional start site of the SV40 early promoter, $A_{n}$ marks the polyadenylation site, $\mathrm{Bgl}$ $\mathrm{II}^{0} / \mathrm{Bam} \mathrm{HI}{ }^{0}$ indicates destroyed restriction sites, and * refers to the position of Arg 109. 


\section{Bradford protein analysis.}

Bradford protein analysis was performed as recommended by Bio-Rad Laboratories. A standard curve was generated by using linearly increasing concentrations of BSA.

\section{Results}

Cloning of OTC cDNA into SV40-based expression vector. To maximize expression of our DNA constructs, we used the expression vector pSV2a containing an SV40 origin of replication (14), enabling electroporated DNA to be amplified as extrachromosomal elements in the permissive cell line Cos1. The scheme used to clone wild type OTC into pSV2a is depicted in Fig. 1. The OTC gene was excised from pHOC3, a cDNA clone derived from the human liver $\lambda$ gt 11 library (2). pHOC3 was partially digested with Eco RI. Linearized species were electrophoresed into an agarose gel, isolated on NA45 paper, and then digested with Hind III. The complete OTC cDNA could be identified as a $1.5-\mathrm{kb}$ fragment and was then purified by gel electrophoresis. The pSV2a vector was prepared by excising the dHFR gene from the parent pSV2a-dHFR by digesting with Bgl II and Hind III and purifying the vector backbone. Then the vector and cDNA were combined with Bam HI-Eco RI adaptors in a three-piece ligation reaction to create pSV2-OTC ${ }^{\mathrm{N}}$.

pSV2-OTC ${ }^{\mathrm{N}}$ was then electroporated into Cos1 cells for transient expression. Subsequent OTC assays revealed that this construct reproducibly expressed high levels of OTC in tissue culture (Table I).

Oligonucleotide-directed mutagenesis. An 850-bp Kpn I fragment spanning the desired mutation site was cloned into M13 at the Kpn I site. Site-directed mutagenesis was performed with the Mutagene Kit (Bio-Rad Laboratories) and using the 18-mer oligonucleotide containing the single base-

Table I. Relative Enzymatic Activities of Mutant and Wild-Type OTCs Indicate that Arg109 Mutation Completely Abolishes OTC Activity in Cos 1

\begin{tabular}{lccc}
\hline & OTC activity & PAP activity & Specific activity \\
\hline & $O D_{490} / h$ & $O D_{595} / h$ & $\mu M /$ min per $m g$ \\
Liver extract & 4.400 & - & 20.63 \\
pSV2-PAP & 0.090 & 1.300 & 0.95 \\
pSV2-OTC & 5.160 & 1.456 & 80.83 \\
pSV2-OTC9 & 0.078 & 1.480 & 0.94 \\
\hline
\end{tabular}

pSV2-OTC ${ }^{\mathrm{N}}$ contains wild-type OTC cDNA, whereas pSV2-OTC9 contains the missense cDNA (Arg109 $\rightarrow$ Gln). pSV2-PAP was used as the negative control for background activity, and rat liver mitochondrial homogenate was used as positive control for OTC activity. Cos 1 cells were electroporated with $80 \mu \mathrm{g}$ OTC cDNA and/or $5 \mu \mathrm{g}$ PAP cDNA and then harvested on the third day of transient expression for enzymatic assays. OTC and PAP activities are defined as the amount of colored product formed per hour, as determined spectrophotometrically. These figures were calculated for $10 \%$ of total culture. Specific activity is defined as micromolar citrulline formed per minute per milligram of total protein, where $\mathrm{OD}_{490}$ of 1.0 is equivalent to $5.5 \mathrm{mM}$ of citrulline. The specific activity was not formally normalized for transfection efficiency since our PAP activities show that transfection efficiencies varied little. pair change corresponding to the Arg 109-to-Gln mutation. Mutants were initially screened by the loss of a unique Xho I site (which includes the four-base Taq I recognition sequence). Subsequent nucleotide sequence analysis confirmed that the $\mathrm{C} \rightarrow \mathrm{T}$ transition is the only change present in the mutant OTC. Finally, the mutagenized Kpn I fragment was cloned back into the wild type pSV2-OTC background for expression in vivo. Restriction analyses demonstrated the integrity of the Kpn I sites and retention of other landmark sites. This mutant construct is designated pSV2-OTC9.

Comparative activities of wild type and mutant OTC. Mutant or wild type pSV2-OTC was electroporated into Cos1 cells for transient expression. pSV2-PAP was cotransfected with the OTC cDNAs as an internal control for transfection efficiency. After a 3-d incubation period, cells were harvested for OTC, PAP, and Bradford assays. Table I shows that the transfection efficiencies of each sample were comparable, as determined from the PAP activities. The wild type OTC $\left(\mathrm{pSV} 2-\mathrm{OTC}^{\mathrm{N}}\right)$ has a high specific activity of $80.83 \mu \mathrm{M} / \mathrm{min}$ per $\mathrm{mg}$. In contrast, the mutant OTC (pSV2-OTC9) has a 100-fold lower specific activity of $0.94 \mu \mathrm{M} / \mathrm{min}$ per $\mathrm{mg}$. Since this level is no higher than that of background (pSV2-PAP), we conclude that the Arg-to-Gln mutation completely abolishes OTC activity.

\section{Discussion}

We have recreated the Arg $109 \rightarrow$ Gln OTC mutation in vitro and have shown that the missense mutation completely abolishes OTC activity in Cos 1 cells. This result confirms that the $\mathrm{C} \rightarrow \mathrm{T}$ transition in the two patients bearing the altered Taq I site is indeed responsible for their OTC-deficient phenotype.

The biochemical impact of this $\mathrm{C} \rightarrow \mathrm{T}$ transition is unclear. Preliminary analysis of mRNA from cells expressing the mutant OTC cDNA driven by the SV40 promoter shows that the basepair alteration does not affect steady state OTC mRNA levels or size. However, no introns are present in this artificial expression vector construct and therefore the effect of the mutation on processing, particularly splicing, of transcripts made from an endogenous cellular OTC gene cannot be assessed. However, the fact that a protein carrying this mutation shows complete loss of enzyme activity suggests a critical role for this residue in the protein without having to invoke an abnormality in splicing as well. In contrast, spf $^{\text {ash }}$, a missense mutation at a different arginine residue, Arg129 of mouse OTC, occurs at an intron-exon boundary and exerts its deleterious effect by unmasking a cryptic splice site (19). The new splice site is used $95 \%$ of the time and leads to the production of a nonfunctional protein. However, when the correct splice site is used, a properly spliced mRNA is translated to a functional protein that contains the amino acid substitution. The spf $^{\text {ash }}$ mutation is therefore strictly a pretranslational mutation which has little or no effect on the protein product.

In light of our preliminary Northern analysis, we have shifted our attention to the analyses of protein stability, assembly, and function.

Interestingly, Arg109 is conserved across the spectrum of bacterial, yeast, and mammalian OTCs (20). Furthermore, it is conserved in Escherichia coli aspartate carbamyl transferase, an enzyme condensing carbamyl phosphate and aspartate that is related to OTC. Sequence comparisons among OTCs of different species have demonstrated that the decapeptide com- 
prising residues $53-62$ is highly conserved $(21-24)$. The core sequence ser-thr-arg-thr-arg contained within the decapeptide has been shown to bind phosphates in several other proteins (25), and has been demonstrated to be highly reactive in bovine OTC (26). Thus, it is speculated that this region of OTC may be involved in binding carbamyl phosphate. Similarly by sequence comparison, the hexapeptide phe-leu-his-cys-leu-pro (residues 268-273) appears to be a highly conserved sequence among all OTCs examined. It has been shown that chemical modification of this conserved cysteine residue in bovine and bacterial OTCs by 5,5'-dithiobis (2-nitrobenzoic acid) results in total loss of affinity for ornithine (27-29). This hexapeptide is therefore believed to reside in the site of ornithine binding.

Although the highly conserved Arg 109 is contiguous with neither the concensus decapeptide nor the hexapeptide, one cannot exclude the possibility that the residue could be at either active site in the three-dimensional protein. The effect of Arg 109 could thus be a loss of catalytic potential caused by the inability to bind ligand. Whether the mutant protein could bind either substrate will be studied by affinity chromatography using ligand-coupled matrices. X-ray crystallography and photoaffinity-labeling experiments would also be needed to clarify these points.

A second possible effect of the missense mutation may be on trimer assembly. There is, as yet, no data on sequence requirements for trimer formation. Our approach to determine what effect Arg109 has on protein assembly will involve immunoblot analysis of native vs. denatured proteins. Finally, there are the possibilities that the mutation could affect protein conformation and/or turnover. Whatever the structural effect, this naturally occurring mutation illustrates the lethality of altering a residue that has been so rigidly preserved by evolution. Finding the molecular role of this residue would further our understanding of OTC enzymology.

\section{Acknowledgments}

We would like to thank Donna Sosnoski and Karen Yadvish for their technical assistance in tissue culture.

Dr. Robert Nussbaum is an associate investigator in the Howard Hughes Medical Institute, and Jeannie Lee is a predoctoral student supported by the Medical Scientist Training Program.

\section{References}

1. Hata, A., T. Tsuzuki, K. Shimada, M. Takiguchi, M. Mori, and I. Matsuda. 1988. Structure of the human ornithine transcarbamylase gene. J. Biochem. (Tokyo). 103:302-308.

2. Maddalena, A., J. E. Spence, W. E. O'Brien, and R. L. Nussbaum. 1988. Characterization of point mutations in the same arginine codon in three unrelated patients with ornithine transcarbamylase deficiency. J. Clin. Invest. 82:1353-1358.

3. Nussbaum, R. L., B. A. Boggs, A. L. Beaudet, S. Doyle, J. L. Potter, and W. E. O'Brien. 1986. New mutation and prenatal diagnosis in ornithine transcarbamylase deficiency. Am. J. Hum. Genet. 38:149-158.

4. Briand, P., B. Francois, D. Rabier, and L. Cathelineau. 1982. Ornithine deficiencies in human males: kinetic and immunochemical classification. Biochim. Biophys. Acta. 704:100-106.

5. Saheki, T., Y. Imamura, I. Inoue, S. Miura, M. Mori, A. Ohtake, M. Tatibana, N. Katsumata, and T. Ohno. 1984. Molecular basis of OTC deficiency lacking enzyme protein. J. Inherited Metab. Dis. 7:2-8.
6. Rosenberg, L. E., F. Kalousek, and M. D. Orsulak. 1983. Biogenesis of ornithine transcarbamylase in spf ${ }^{\text {ash }}$ mutant mice: two cytoplasmic precursors, one mitochondrial enzyme. Science (Wash. DC). 222:426-428.

7. Veres, G., R. A. Gibbs, S. E. Scherer, and C. T. Caskey. 1986. The molecular basis of the sparse fur mouse mutation. Science (Wash. DC). 237:405-417.

8. Rozen, R., J. E. Fox, W. A. Fenton, A. L. Horwich, and L. E. Rosenberg. 1985. Gene deletion and restriction fragment length polymorphisms at the human ornithine transcarbamylase locus. Nature (Lond.). 313:815-817.

9. Old, J. M., P. L. Briand, S. Purvis-Smith, N. J. Howard, B. Wilcken, J. Hammond, P. Pearson, L. Cathelineau, R. Williamson, and K. E. Davies. 1985. Prenatal exclusion of ornithine transcarbamylase by direct gene analysis. Lancet. i:73-75.

10. Rozen, R., J. E. Fox, A. M. Hack, W. A. Fenton, A. L. Horwich, and L. E. Rosenberg. 1986. DNA analysis for ornithine transcarbamylase deficiency. J. Inherited Metab. Dis. 9:49-57.

11. McClead, R. E., Jr., R. Rozen, J. Fox, L. Rosenberg, J. Menke, R. Bichers, and G. Morrow III. 1986. Clinical applications of DNA analysis in a family with OTC deficiency. Am. J. Med. Genet. 25:513538 .

12. Maniatis, T., E. F. Fritsch, and J. Sambrook. 1982. Molecular Cloning: A Laboratory Manual. Cold Spring Harbor Laboratory, Cold Spring Harbor, NY. 545 pp.

13. Horwich, A. L., W. A. Fenton, K. R. Williams, F. Kalousek, J. P. Kraus, R. F. Koolittle, W. Konisberg, and L. E. Rosenberg. 1984. Structure and expression of a complementary DNA for the nuclear encoded precursor of human mitochondrial ornithine transcarbamylase. Science (Wash. DC). 224:1068-1074.

14. Kadesch, T., and P. Berg. 1986. Effects of the position of the simian virus 40 enhancer on expression of multiple transcription units in a single plasmid. Mol. Cell. Biol. 7:2593-2601.

15. Henthorn, P., P. Zervos, M. Raducha, H. Harris, and T. Kadesch. 1988. Expression of a human placenta alkaline phosphatase gene in transfected cells: use as a reporter for studies of gene expression. Proc. Natl. Acad. Sci. USA. 85:6342-6346.

16. Gluzman, Y. 1981. SV40-transformed simian cells support the replication of early SV40 mutants. Cell. 23:175-182.

17. Chu, G., H. Hayakawa, and P. Berg. 1987. Electroporation for the efficient transfection of mammalian cells with DNA. Nucleic Acids Res. 15:1311-1326.

18. Farkas, D. H., C. J. Skomra, G. R. Anderson, and R. G. Hughes. 1987. In situ staining procedure for the detection of ornithine transcarbamylase activity in polyacrylamide gels. Anal. Biochem. $160: 421-428$.

19. Hodges, P. E., and L. E. Rosenberg. 1988. The $\mathrm{spf}^{\text {ash }}$ mutation of the mouse ornithine transcarbamylase gene affects splice donor site choice and results in two mutant proteins. Am. J. Hum. Genet. 43:A186. (Abstr.)

20. Huygen, R., M. Crabeel, and N. Glansdorff. 1987. Nucleotide sequence of the arg3 gene of the yeast Saccharomyces cerevisiae encoding ornithine carbamoyltransferase: comparison with other carbamoyltransferases. Eur. J. Biochem. 166:371-377.

21. Bencini, D. A., J. E. Houghton, T. A. Hoover, K. F. Foltermann, J. R. Wild, and G. A. O'Donovan. 1983. The DNA sequence of arg1 from Escherichia coli K12. Nucleic Acids Res. 11:8509-8518.

22. Hoover, T. A., W. D. Roof, D. F. Foltermann, G. A. O'Donovan, D. A. Bencini, and J. R. Wild. 1983. Nucleotide sequence of the structural gene (pyrB) that encodes the catalytic polypeptide of aspartate transcarbamylases of Escherichia coli. Proc. Natl. Acad. Sci. USA. 30:2462-2466.

23. Van Vliet, F., R. Cunin, A. Jacobs, J. Piette, D. Gigot, M. Lauwereys, A. Pierard, and N. Glansdorff. 1984. Evolutionary divergence of genes for ornithine and aspartate carbamoyltransferases. 
Complete sequence and mode of regulation of the Escherichia coli argF gene: comparison with argF and argI and pyrB. Nucleic Acids Res. 12:6277-6289.

24. Kraus, J. P., P. E. Hodges, L. L. Williamson, A. L. Horwich, F. Kalousek, K. R. Williams, and L. E. Rosenberg. 1985. A cDNA clone for the precursor of rat mitochondrial ornithine transcarbamylase: comparison of rat and human leader sequences and conservation of catalytic sites. Nucleic Acids Res. 13:943-952.

25. Schulz, G. E., and R. H. Schirmer. 1979. Principles of Protein Structure. Springer Publishing Company, New York. 226.

26. Marshall, M., and P. P. Cohen. 1980. Evidence of an exceptionally reactive arginyl residue at the binding site of carbamoyl phos- phate in bovine ornithine transcarbamylase. J. Biol. Chem. 255:73017305.

27. Marshall, M., and P. P. Cohen. 1980. Ornithine transcarbamylases: ordering of S-cyano peptides and location of characteristically reactive cysteinyl residues within the sequence. J. Biol. Chem. 255:7287-7305.

28. Marshall, M., and P. P. Cohen. 1980. The essential sulfhydryl group of ornithine transcarbamylases: reaction with anionic, aromatic disulfides and properties of its cyano derivative. J. Biol. Chem. 255:7287-7290.

29. Marshall, M., and P. P. Cohen. 1980. The essential sulfhydryl group of ornithine transcarbamylases: $\mathrm{pH}$ dependence of the spectra of its 2-mercuri-5-nitrophenol derivative. J. Biol. Chem. 255:7296-7300. 